\title{
Synergistic effects of perfluoroalkyl acids mixtures with J-shaped concentration-responses on viability of a human liver cell line
}

\author{
Jiayue $\mathrm{Hu}^{\mathrm{a}, \mathrm{b}}$, Juan $\mathrm{Li}^{\mathrm{c}}$, Jianshe Wang ${ }^{\mathrm{a}}$, Aiqian Zhang ${ }^{\mathrm{c}}$, Jiayin Dai ${ }^{\mathrm{a}, *}$ \\ ${ }^{a}$ Key Laboratory of Animal Ecology and Conservation Biology, Institute of Zoology, Chinese Academy of Sciences, Beijing 100101, PR China \\ ${ }^{\mathrm{b}}$ Graduate School of the Chinese Academy of Sciences, Beijing 100080, PR China \\ ' State Key Laboratory of Environmental Chemistry and Ecotoxicology, Research Center for Eco-Environmental Sciences, Chinese Academy of Sciences, Beijing 100085, PR China
}

\section{H I G H L I G H T S}

- The first report of PFAAs mixture effects of environmentally relevant contaminants.

- The inhibitory effect of PFAAs increased with the elongation of the carbon chain.

- Volume and solute accessible surface area dominated the cytotoxic effects of PFAAs.

- Synergistic effects occurred under $\mathrm{IC}_{0}$, $\mathrm{IC}_{10}$, and $\mathrm{IC}_{50}$ in binary mixtures.

\section{A R T I C L E I N F O}

\section{Article history:}

Received 8 December 2012

Received in revised form 10 July 2013

Accepted 13 July 2013

Available online 12 August 2013

\section{Keywords:}

Perfluorinated compounds

Non-monotonic concentration-response

Joint effect

Synergism

Quantitative structure-activity relationship
G R A P H I C A L A B S T R A C T

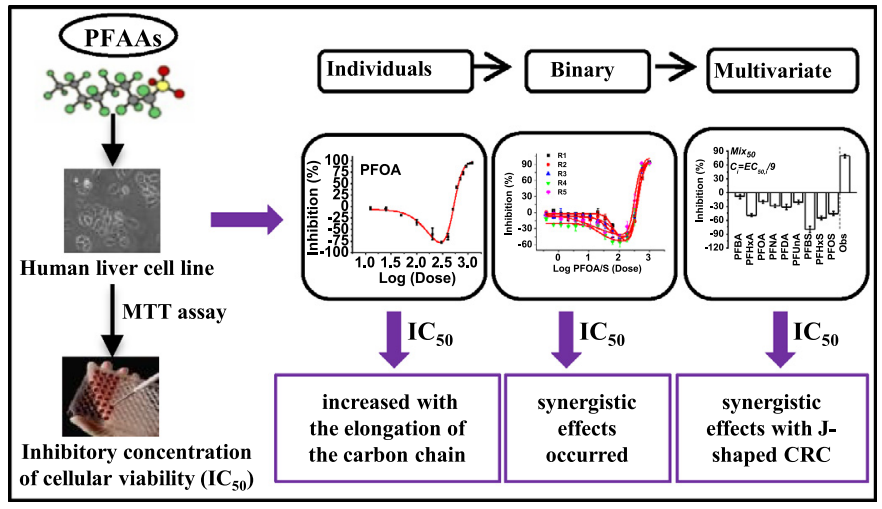

\begin{abstract}
A B S T R A C T
Some perfluoroalkyl acids (PFAAs) are highly persistent and bioaccumulative, resulting in their broad coexisting distribution in humans and the environment. Our aim was to investigate the individual and joint effects of PFAAs on cellular viability of a human liver cell line (HL-7702) using the MTT assay. Equipartition ray design and equivalent-effect concentration ratio (EECR) mixtures were used to investigate the binary and multiple effects of PFAAs, respectively. All tested PFAAs mixtures and the individuals (except perfluorododecanoic acid (PFDoDA) and perfluorotetradecanoic acid (PFTeDA)) showed obvious non-monotonic J-shaped concentration-response curves (CRC) on HL-7702. The inhibitory effect of individual PFAAs increased with the elongation of the carbon chain and was dominated by their molecular volume. The three binary mixtures (PFOA/S, PFHxA/S and PFBA/S) showed that synergistic effects occurred under effective inhibitory concentrations (IC) of $\mathrm{IC}_{0}$, $\mathrm{IC}_{10}$, and $\mathrm{IC}_{50}$ in mixtures, while for $\mathrm{IC}_{-20}$ the synergistic effect only occurred under higher PFSA proportion in mixtures. Furthermore, EECR mixtures of the nine individual PFAAs with J-shaped CRC also showed synergistic effects. However, mixtures of the eleven individual PFAAs including those with S-shaped CRC resulted in partial addition effects on HL-7702. Our results indicated that the individual stimulatory responses of HL-7702 to PFAA may produce adverse effects in mixtures at relevant dose levels.
\end{abstract}

(c) 2013 Elsevier Ltd. All rights reserved.

\footnotetext{
* Corresponding author. Tel.: +86 10 64807185; fax: +86 1064807099 .

E-mail address: daijy@ioz.ac.cn (J. Dai).
}

\section{Introduction}

Perfluoroalkyl acids (PFAAs), including perfluoroalkyl carboxylic acids (PFCAs) and perfluoroalkane sulfonic acids (PFSAs) (Buck et al., 2011), are a family of perfluorinated chemicals consisting 
typically of a 4-14 carbon length backbone (Kowalczyk et al., 2012; Filipovic et al., 2013). Many PFAAs, which are highly resistant to degradation and are environmentally persistent (Conder et al., 2008), have been widely used in commercial and industrial products (Renner, 2001). Because of their wide industrial application and common global use in consumer products in recent decades, PFAAs have been detected in the liver, fat, and serum of wildlife (De Silva and Mabury, 2006), and in human serum and blood (Kannan et al., 2004), breast milk (Kärrman et al., 2006), and umbilical cord blood (Apelberg et al., 2007). Perfluorooctanoic acid (PFOA) and perfluorooctane sulfonic acid (PFOS) both dominate in human serum (Lau et al., 2004) and a number of PFSAs and PFCAs of varying chain length (C4-C14) have also been detected in human body fluids, though at lower levels (Olsen et al., 2005). Studies have shown that PFAAs with longer fluorinated carbon chains ( $>7$ fluorinated carbons) mainly accumulate in the liver (Lau et al., 2004; Houde et al., 2006) and thus lead to both in vivo and in vitro hepatic morphological and biochemical changes in laboratory animals (Seacat et al., 2003; Son et al., 2008; Wolf et al., 2008; Zhang et al., 2008; 2012; Albrecht et al., 2013). Although these studies suggest that the peroxisome proliferators activated receptor $\alpha$ (PPAR $\alpha$ )-dependent mode of action proposed for hepatic tumor induction in rodents is not relevant to humans (Bjork and Wallace, 2009), recent epidemiological data found significant positive associations between PFOA and PFOS concentrations and all lipid outcomes (except high density lipoprotein-cholesterol), with a relatively lower serum level of PFOA (median, $27 \mathrm{ng} / \mathrm{mL}$ ) and PFOS (median, $20 \mathrm{ng} / \mathrm{mL}$ ) in 46,294 community residents from a West Virginian chemical plant (C8 Health Project) (Steenland et al., 2009). In addition, researchers have found a linear association between PFOA/PFOS serum concentrations and alanine transferase (a marker of hepatocellular injury) (Gallo et al., 2012). Thus, the ubiquitous presence and persistence of PFAAs in the environment and within the human body have led to efforts to understand adverse effects that may be associated with exposure, in particular scientific and regulatory concerns.

While previous studies have investigated the individual toxic effects and mechanisms of PFAAs, few studies have assessed the combined effects of PFAAs in multi-component mixtures. Pollutants always coexist in the environment and mixtures represent the prevailing form of environmentally occurring contaminants (Guida et al., 2008). The interactions between different chemical components in a mixture may result in either antagonistic or synergistic combined effects rather than the additive effect only as expected from the toxicity and mode of action of each individual compound. Various PFAAs commonly coexist in the environment as well as in organisms (Kannan et al., 2004; De Silva and Mabury, 2006). The uncertainty regarding the combined effects and interactions between different PFAAs has hampered the hazard identification and risk assessment of PFAAs. Therefore, studies investigating the combined effects of various PFAAs and the interaction among chemicals under co-exposure are required.

The 3-(4,5-dimethylthiazol-2-yl)-2,5-diphenyltetrazolium bromide (MTT) assay measures cellular metabolic activity via reduced nicotinamide-adenine dinucleotide phosphate (NAD(P)H)-dependent cellular oxidoreductase enzymes and reflects the number of viable cells. This assay has attractive features including easy handling, high sensitivity, short assay time, and non-radioactivity. Therefore, MTT assay has been widely used to measure cytotoxicity or cytostatic activity of potential medicinal agents and toxic chemicals (Berridge et al., 2005).

In the present study, eleven straight-chain PFAAs with backbones from 4 to 14 carbon atoms in length were used to explore potential toxicity to a human liver cell line (HL-7702). The inhibitory concentration of cellular viability $\left(\mathrm{IC}_{x}\right.$ ) by individual PFAAs and their mixtures as endpoints were detected using the MTT assay. The direct equipartition ray (EquRay) was used to characterize the concentration composition of various binary mixtures (Dou et al., 2011) and equivalent-effect concentration ratio (EECR) to analyze multi-component mixtures (Ge et al., 2011; Liu et al., 2012). The aims of this study were to (a) investigate the concentration-response relationships of individual PFAAs and compare their toxic effects on human liver cell line; (b) explore their structural characteristics of individual PFAAs in relation to their inhibitory effects on human liver cell line using the quantitative structureactivity relationship (QSAR) method; and (c) examine the joint effects of binary and multiple mixtures of PFAAs on human liver cell line. Our research will improve the understanding how they interact with each other using human liver cell line as an alternative in vitro test.

\section{Materials and methods}

\subsection{Chemicals}

Perfluorobutyric acid (PFBA), perfluorohexanoic acid (PFHxA), PFOA, perfluorononanoic acid (PFNA), perfluorodecanoic acid (PFDA), perfluoroundecanoic acid (PFUnDA), PFDoDA, PFTeDA, potassium perfluorobutane sulfonate (PFBS) and potassium perfluorohexane sulfonate (PFHxS) were purchased from Sigma (St. Louis, MO, USA), and all information including the purity ( $>95-$ $98 \%$ ) of the chemicals is given in the supporting information (Table S1). Chemical solutions were made as stock solutions and prepared fresh on the day of treatment. We dissolved PFNA, PFDA, PFUnDA, PFDoDA, PFTeDA, PFBS, PFHxS, and PFOS in ethanol and the highest final concentration of ethanol was less than $0.5 \%$ in the stock solutions. Solvents at the highest concentration $(0.05 \%)$ in working solutions did not cause cytotoxicity to the human liver cell line. All other PFAAs were dissolved directly in serum-free RPMI-1640 medium. Both MTT and dimethyl sulfoxide (DMSO) were purchased from Sigma-Aldrich (USA). Fetal calf serum was obtained from Hangzhou Sijiqing Co., Ltd. (Hangzhou, China), and RPMI-1640 medium was obtained from Gibco (Glasgow, UK).

\subsection{Cell culture, treatment and MTT assay}

The immortalized fetal human liver cell line (HL-7702) was obtained from the Shanghai Institute of Cell Biology, Chinese Academy of Sciences. They were cultured in RPMI-1640 complete culture medium in a humidified atmosphere of $5 \% \mathrm{CO}_{2}$ at $37{ }^{\circ} \mathrm{C}$. For experimental purposes, cells were trypsinized and seeded on 96-well plates at a density of $1 \times 10^{4}$ cells/well in appropriate culture medium. Cells were allowed to attach for $24 \mathrm{~h}$ before the experiments were performed. Afterwards cells were treated with PFAAs at a range of concentrations (Table S1) for $48 \mathrm{~h}$. Cellular viability as the endpoint was determined using MTT assay $(5 \mathrm{mg} / \mathrm{mL})$ dissolved in PBS and stored at $4{ }^{\circ} \mathrm{C}$. After an incubation period, $20 \mu \mathrm{L}$ of MTT was added to each well followed by incubation at $37^{\circ} \mathrm{C}$ for $4 \mathrm{~h}$. Supernatants were then removed from the plates and the produced formazan was dissolved in $150 \mu \mathrm{L}$ of DMSO and measured at $550 \mathrm{~nm}$ using a spectrophotometer (BioTek, Synergy H1 Hybrid Microplate Reader, USA). The inhibition of cellular viability (\%) was calculated as follows: Inhibition $(\%)=\left(1-\mathrm{OD}_{\text {treated }}\right)$ $\left.\mathrm{OD}_{\text {untreated }}\right) \times 100 \%$.

\subsection{Binary mixtures of PFAAs and direct equipartition ray design}

We selected PFCAs (PFOA, PFHxA, and PFBA) and PFSAs (PFOS, PFHxS, and PFBS) as the binary mixture components using EquRay procedure as described previously (Dou et al., 2011). Briefly, in the two-dimension $(X-Y)$ concentration plane constructed by the PFCA 
and PFSA axes, the $\mathrm{IC}_{50}$ point at the $X$-axis (e.g. PFOS) was connected to the $\mathrm{IC}_{50}$ point at the $Y$-axis (e.g. PFOA) to form a line segment on which five equidistance points were set. From the origin (O), five rays ( $R 1, R 2, R 3, R 4$, and $R 5$ ) were drawn through five equidistance points (supporting information, Fig. S1). Twelve concentration points $\left(x_{i}, y_{i}, i=1,2, \ldots, 12\right)$ on each of the five rays were then designed using a proper dilution factor (fray) where the ratio of $x / y$ was fixed for twelve concentration points of a ray. Finally, the cellular viability of the points (binary mixtures) of HL-7702 were determined using the above MTT procedure to build five mixture concentration-response curves ( $\mathrm{m}-\mathrm{CRCs}$ ), where one ray corresponded to one $\mathrm{m}-\mathrm{CRC}$.

\subsection{Experimental design for multi-component mixtures}

Eleven or nine individual PFAAs were selected to study the joint effect of various combinations of PFAAs mixtures that may coexist in the environment. Four EECR mixtures $\left(\mathrm{Mix}_{50}, \mathrm{Mix}_{10}, \mathrm{Mix}_{0}\right.$, and Mix $_{-20}$ ratio) were designed as described previously (Ge et al., 2011). In the mixtures, the concentration percentages of various PFAAs were percentages of their individual PFAA's $\mathrm{IC}_{50}, \mathrm{IC}_{10}, \mathrm{IC}_{0}$, and $\mathrm{IC}_{-20}$ to the total concentration of the mixtures, respectively. $\mathrm{The} \mathrm{Mix}_{0}$ was especially designed for the non-monotonic J-shaped CRCs to compare the joint effect of PFAAs with J-shaped CRCs to those with classical S-shaped CRCs. To compare the joint effect of those EECRs, mixtures with $\mathrm{IC}_{0, i}$ concentrations of individual PFAAs (each PFAA induces no inhibitory effect singly) were also designed.

\subsection{Mixture toxicity evaluation}

Three indicators were proposed to quantify and analyze the joint effects, specifically Toxic Unit (TU), Additive Index (AI), and Mixture Toxicity Index (MTI) (Xu and Nirmalakhandan, 1998; Koutsaftis and Aoyama, 2007). The expected toxicity (toxic strength) of the mixtures (based on the non-additive hypothesis) was expressed as toxic units (TU). The toxicity of the mixtures $(M)$, conducted similarly to the single-chemical tests, was described by the sum of TUs, according to the equation:

$\mathrm{TU}_{i}=c_{i} / \mathrm{IC}_{50, i} ; M=\sum \mathrm{TU}_{i} ; M_{0}=M / \max \left(\mathrm{TU}_{i}\right) ;$

where $c_{i}$ is the concentration of individual chemical $i$; $\mathrm{IC}_{50, i}$ is the $\mathrm{IC}_{50}$ of individual chemical $i$; and $\mathrm{IC}_{50}$ is $50 \%$ effective concentration. Further, $M=1$ : simple addition; $M>M_{0}$ : antagonism; $M<1$ : synergism; $M=M_{0}$ : independent action; and $M_{0}>M>1$ : partial addition.

The additive index (AI) and the mixture toxic index (MTI) were calculated according to the equation:

$\mathrm{AI}=1 / M-1(M \leq 1) ; \mathrm{AI}=1-M(M>1)$

where $\mathrm{AI}<0$ : antagonism; and $\mathrm{AI}>0$ : synergism

MTI $=1-\log M / \log M_{0}$

where $\mathrm{MTI}<0$ : antagonism; MTI > 1: synergism; $\mathrm{MTI}=0$ : independent action; and $1>$ MTI $>0$ : partial addition.

\subsection{Calculation of molecular descriptors}

Molecular descriptors were obtained using Dragon 6.0 (Talete srl, Milano, Italy). There was no need to attempt all molecular descriptors provided by Dragon 6.0 because the tested compounds shared a common skeleton. High structural similarity inevitably resulted in identical or zero values for many kinds of descriptors, and the descriptors with lower standard deviations were excluded. Only molecular connectivity indices (MCIs) and solvation connectivity indices for tested compounds were maintained, respectively.
The relationship between biological activity data and chemical structure descriptors was obtained by stepwise linear regression with a confidence interval of $95 \%$ (Zhu et al., 2009). The parameters included in the regression equation are listed in Table S2.

\subsection{Calculation of inhibitory concentration $\left(I_{50}\right)$ and data analysis}

To calculate half-maximal inhibitory concentration $\left(\mathrm{IC}_{50}\right)$ as well as the $\mathrm{IC}_{-20}$ (20\% stimulatory effect concentration), IC $\mathrm{IC}_{10}$, and $\mathrm{IC}_{5}$ values in individual PFAAs, binary mixtures and multi-component mixtures, BiPhasic and DoseResp models were used to fit the J-shaped and S-shaped curves using Origin pharmacology in Origin8.0 (Origin Lab Corporation, Northampton, USA), respectively. The J-shaped CRC is described by Eq. (1) derived from a BiPhasic equation:

$y=A_{\min }+\frac{\left(A_{\max 1}-A_{\min }\right)}{1+10^{\left(\left(x-x 0_{-} 1\right) * h 1\right)}}+\frac{\left(A_{\max 2}-A_{\min }\right)}{1+10^{\left(\left(x 0 \_2-x\right) * h 2\right)}}$

where $A_{\max 1}$ and $A_{\max 2}$ are the first and second top asymptotes, $x_{0 \_}$and $x_{0 \_}$are the first and second top medians, $h_{1}$ and $h_{2}$ are slopes, and $A_{\min }$ is the bottom.

The S-shaped CRC is described by Eq. (2) derived from a DoseResp equation:

$y=A 1+\frac{A 2-A 1}{1+10^{(\mathrm{LOG} x 0-x) p}}$

where $A 1$ and $A 2$ are the bottom and top asymptotes, $x 0$ is the center point of the curve and $\mathrm{p}$ is the hill slope.

Regression analysis was performed using non-linear leastsquares fit. The higher the coefficient of determination $\left(R^{2}\right)$ and the lower the Chi-Sqr, the better was the fit. As a quantitative measure of uncertainty, the observation-based 95\% confidence interval was also determined.

\section{Results and discussion}

\subsection{Concentration-response relationships of individual PFAAs}

To investigate the concentration response relationships of individual PFAAs, we exposed HL-7702 cells to PFAAs for $48 \mathrm{~h}$, with the concentration-response curve (CRC) of the eleven PFAAs presented in Fig. 1. In the present study, nine individual PFAAs (C4-C11) showed J-shaped non-monotonic CRCs and two long-chain PFCAs (C12, C14) showed S-shaped CRCs. The J-shaped CRCs were fitted with the biphasic concentration-response models while the monotonically S-shaped CRCs were fitted using the DoseResp concentration-response models (Fig. 1). The fitted CRC models and resulting parameters including half-inhibitory concentration ( $\left.\mathrm{IC}_{50}\right)$, as well as $\mathrm{IC}_{0}, \mathrm{IC}_{10}$, and $\mathrm{IC}_{-20}$ are listed in Table 1 . Results showed that PFNA presented the highest stimulatory effect $\left(E_{m}=-85 \%\right)$ at $1.36 \times 10^{-4} \mathrm{M}$ (maximal stimulatory effect concentration) and PFHXS had a wide zone ranging from $5.2 \times 10^{-5} \mathrm{M}$ to $6.7 \times 10^{-4} \mathrm{M}$, with a maximum stimulatory effect of $E_{m}=-54 \%$. The values of $\mathrm{IC}_{50}$ ranged from $9.23 \times 10^{-3} \mathrm{M}$ (PFBA) to $3.47 \times 10^{-5} \mathrm{M}$ (PFTdDA), indicating that PFTdDA was the most toxic compound. With the same carbon chain length, PFSAs showed higher stimulatory effects than PFCAs and the values of IC $_{-20}$ for PFBA, PFHxA, and PFOA were 45.67, 15.76, and 6.37-fold higher, respectively, to corresponding PFSAs.

\subsection{Quantitative structure-activity relationship of PFAAs}

To further explore the relationship of PFAA chain length with inhibitory effects, we performed a linear regression, whereby we 

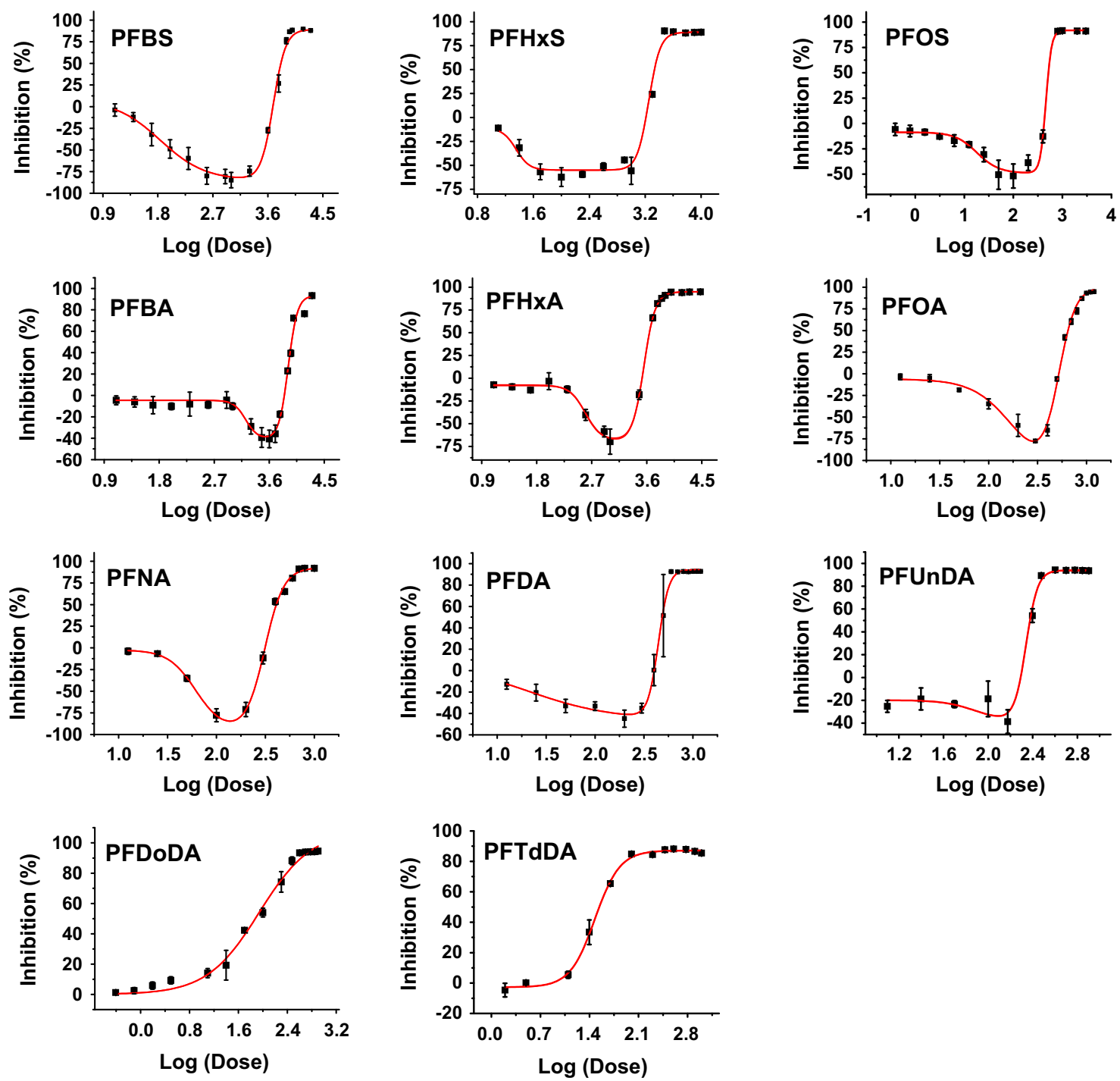

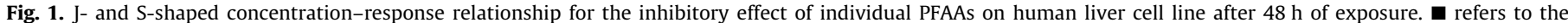
experimental data, the solid line $(-)$ is the fitted concentration response curve (CRC).

Table 1

Concentration-response models of individual PFAAs on the inhibitory effect in human liver cell line at $48 \mathrm{~h}$.

\begin{tabular}{|c|c|c|c|c|c|c|c|c|c|}
\hline Abb. & Model & Chi-Sqr & $R^{2}$ & $\mathrm{IC}_{-20}$ & $\mathrm{IC}_{0}$ & $\mathrm{IC}_{10}$ & $\mathrm{IC}_{50}$ & ${ }^{0} \chi$ & $X_{0 \text { sol }}$ \\
\hline PFBA & BiPhasic & 30.9629 & 0.9809 & $1.53 \times 10^{-3}$ & $7.03 \times 10^{-3}$ & $7.45 \times 10^{-3}$ & $9.23 \times 10^{-3}$ & 11.077 & 4.992 \\
\hline PFHxA & BiPhasic & 7.9350 & 0.9979 & $2.60 \times 10^{-4}$ & $3.33 \times 10^{-3}$ & $3.51 \times 10^{-3}$ & $4.40 \times 10^{-3}$ & 16.077 & 6.406 \\
\hline PFOA & BiPhasic & 38.7675 & 0.9902 & $7.14 \times 10^{-5}$ & $5.21 \times 10^{-4}$ & $5.42 \times 10^{-4}$ & $6.47 \times 10^{-4}$ & 21.077 & 7.820 \\
\hline PFNA & BiPhasic & 27.4615 & 0.9933 & $3.84 \times 10^{-5}$ & $3.14 \times 10^{-4}$ & $3.29 \times 10^{-4}$ & $4.09 \times 10^{-4}$ & 23.577 & 8.527 \\
\hline PFDA & BiPhasic & 17.7496 & 0.9951 & $2.20 \times 10^{-5}$ & $4.02 \times 10^{-4}$ & $4.19 \times 10^{-4}$ & $4.85 \times 10^{-4}$ & 26.077 & 9.234 \\
\hline PFUnDA & BiPhasic & 44.1727 & 0.9874 & $1.54 \times 10^{-5}$ & $1.96 \times 10^{-4}$ & $2.04 \times 10^{-4}$ & $2.37 \times 10^{-4}$ & 28.577 & 9.941 \\
\hline PFDoDA & DoseResp & 9.5947 & 0.9928 & - & - & $9.19 \times 10^{-6}$ & $7.51 \times 10^{-5}$ & 31.077 & - \\
\hline PFTdDA & DoseResp & 3.6815 & 0.9975 & - & $7.28 \times 10^{-6}$ & $1.44 \times 10^{-5}$ & $3.47 \times 10^{-5}$ & 36.077 & - \\
\hline PFBS & BiPhasic & 38.7976 & 0.9918 & $3.35 \times 10^{-5}$ & $4.81 \times 10^{-3}$ & $5.07 \times 10^{-3}$ & $6.49 \times 10^{-3}$ & 15.207 & 8.578 \\
\hline PFHxS & BiPhasic & 49.2899 & 0.9892 & $1.65 \times 10^{-5}$ & $1.62 \times 10^{-3}$ & $1.71 \times 10^{-3}$ & $2.14 \times 10^{-3}$ & 20.207 & 9.993 \\
\hline PFOS & BiPhasic & 170.0378 & 0.9608 & $1.12 \times 10^{-5}$ & $4.23 \times 10^{-4}$ & $4.41 \times 10^{-4}$ & $5.16 \times 10^{-4}$ & 25.207 & 11.407 \\
\hline
\end{tabular}

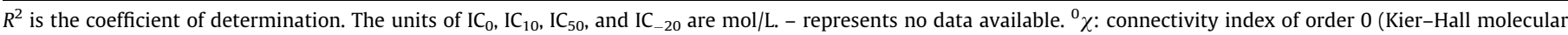
connectivity indices), $X_{0 \text { sol }}$ : solvation connectivity index of order zero.

defined the number of carbon atoms in a backbone as $N_{m}$. A statistically significant negative linear correlation was observed between $\log \mathrm{IC}_{50}$ and $N_{m}\left(R^{2}=0.972\right)$ for the eleven PFAAs, indicating that the longer the perfluorinated chain, the stronger the inhibitory effect (Fig. 2A).
To explore the determinant factors of the inhibitory effect of PFAAs, we calculated their chemical descriptors and developed a QSARs model using stepwise linear regression (Li et al., 2010), in which molecular connectivity index of order zero $\left({ }^{0} \chi\right)$ entered the final model. 

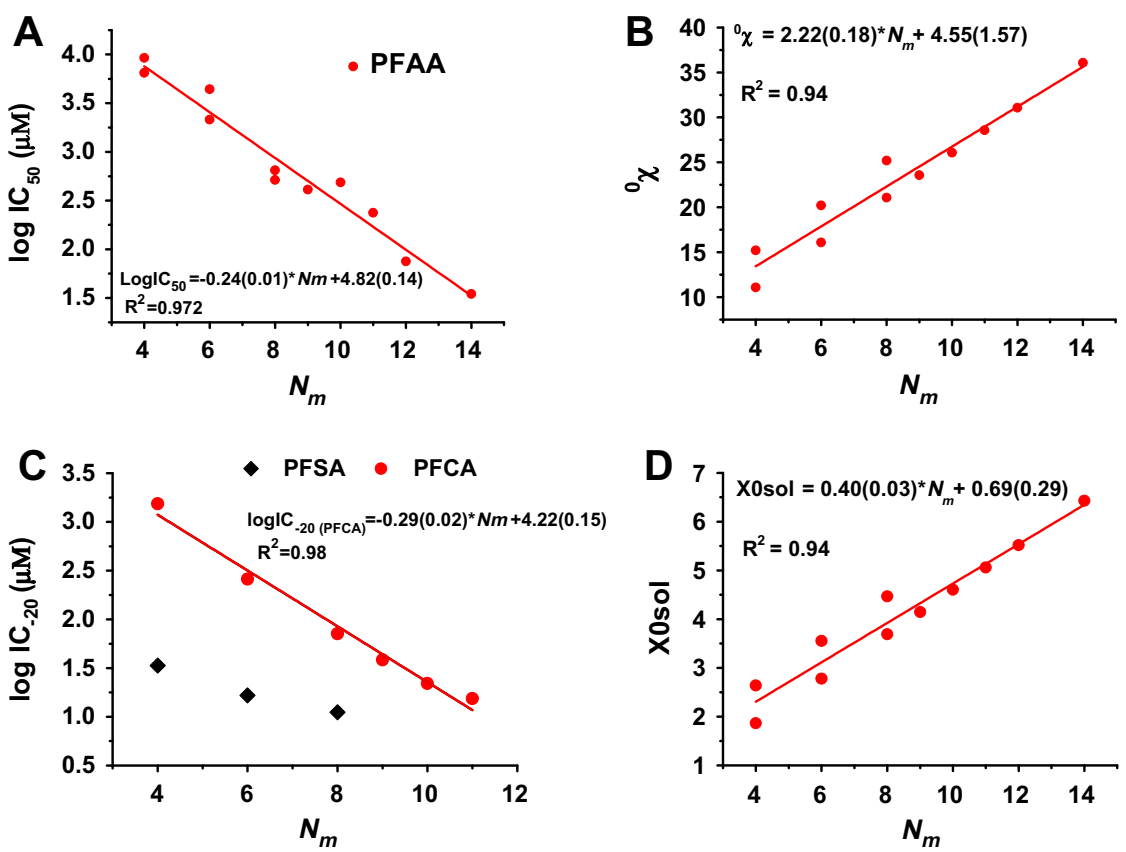

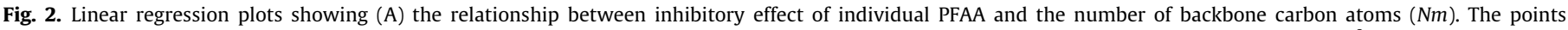

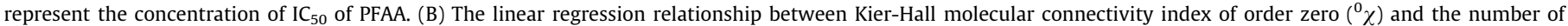

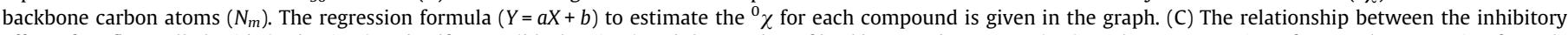

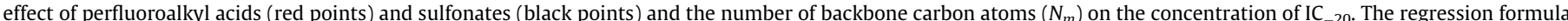

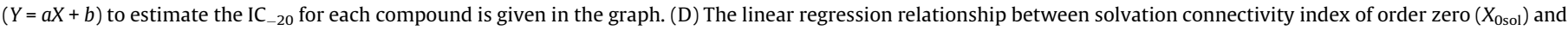
the number of backbone carbon atoms $\left(N_{m}\right)$. The regression formula $(Y=a X+b)$ to estimate the $X_{0 \text { sol }}$ for each compound is given in the graph.

$-\log \mathrm{IC}_{50}=0.103( \pm 0.007) \times{ }^{0} \chi+0.770( \pm 0.163)$

$n=11, R^{2}=0.963, R_{\text {adj }}^{2}=0.959, \mathrm{SE}=0.157, F=233.278, P=0.000$

where $n$ is the number of observations, $R$ is the correlation coefficient, $\mathrm{SE}$ is the standard error of estimates, $F$ is the $F$-test value, and $p$ is the significance level of the whole equation. A statistically significant linear correlation between $\log I \mathrm{C}_{50}$ and ${ }^{0} \chi$ indicated that $\mathrm{IC}_{50}$ could be predicted by models based on molecular connectivity descriptors (Table S2). In addition, ${ }^{0} \chi$ was positively correlated with $N_{m}\left(R^{2}=0.94, n=11\right)$ for the PFAAs in our study (Fig. 2B). Generally, a Kier-Hall molecular connectivity index of order zero $\left({ }^{0} \chi\right)$ symbolizes molecular size or bulky volume related property. These results showed that the volume and solute accessible surface area dominated the inhibitory effect of tested PFAAs and that protein affinity might play a key role in toxicity.

Previous research has shown that the expression of gene targets related to cell injury in primary rat hepatocytes caused by PFAAs is related to the length of each compound's carbon chain and concentration (Bjork and Wallace, 2009). In addition, transthyretin binding affinity of PFAAs is associated with the length of each compound's carbon chain (Weiss et al., 2009). Our results indicated that the longer the perfluorinated chain, the stronger was the inhibitory effect on cellular viability. The model developed by simple descriptors based on molecular connectivity indices of the compounds could predict the inhibitory effect of PFAAs.

As for the stimulation effect, the relationship between $\log _{\mathrm{I}} \mathrm{C}_{-20}$ and $N_{m}$ was different from that of the inhibition effect. For IC -20 , a negative correlation was observed in six PFCAs $\left(R^{2}=0.981\right)$ when shielding the PFSAs (Fig. 2C). Stimulation reached maximum at $N_{m}$ of 9-10, while PFDoDA and PFTdDA showed no stimulation effect under the experimental concentrations $\left(3.90 \times 10^{-7} \mathrm{M}\right.$ and $1.56 \times 10^{-6} \mathrm{M}$, respectively). The decrease in activity enhancement caused by increases in $N_{m}$ helped to differentiate perfluorinated alkyl sulfonic acids from perfluorinated alkyl carboxylic acids. With the same carbon chain length, the PFSAs had lower IC -20 than PFCAs (Table 1 and Fig. 2C), which suggested that the stimulatory effect was governed, to some degree, by the contribution of functional groups as well as carbon chain length.

To gain further information on the stimulation effect of the low dose effect in our test system, a structure-based model study was performed. Solvation connectivity index of order zero $\left(X_{0 \text { sol }}\right)$ entered the final model by stepwise linear regression.

$-\log \mathrm{IC}_{-20}=0.348( \pm 0.035) \times \chi_{\text {osol }}+1.331( \pm 0.304)$

$n=9, R^{2}=0.935, R_{a d j}^{2}=0.925, \mathrm{SE}=0.192, F=100, P=0.000$

In Eq. (4), a statistically significant linear correlation between $\log \mathrm{CC}_{-20}$ and $X_{0 \text { sol }}$ indicated that IC ${ }_{-20}$ could be predicted by solvation connectivity index based models (Table S2). In addition, $X_{0 \text { sol }}$ was positively correlated with chain length $\left(R^{2}=0.94, n=11\right)$ for the PFAAs (Fig. 2D). As a solvation connectivity index, $X_{0 \text { sol }}$ could be considered as the entropy of solvation, which indicates dispersion interactions in solutions (Zhao et al., 2008). Our results showed that both non-polar interactions, as indicated by $N_{m}$ and electrostatic-like forces provided by acidic groups, might predominate in the stimulation effect.

\subsection{Non-monotonic concentration-response relationships of binary mixtures}

Most reports on non-monotonic concentration-response relationships have focused on individual chemicals, with few studies exploring the joint effect of multiple chemical mixtures (Ge et al., 2011; Wang et al., 2011; Keiter et al., 2012). We first selected PFOA and PFOS to investigate the joint effects of binary mixtures because of their predominant detection in human serum and liver. Two other binary mixtures, namely PFHxA-PFHxS and PFBA-PFBS, were 

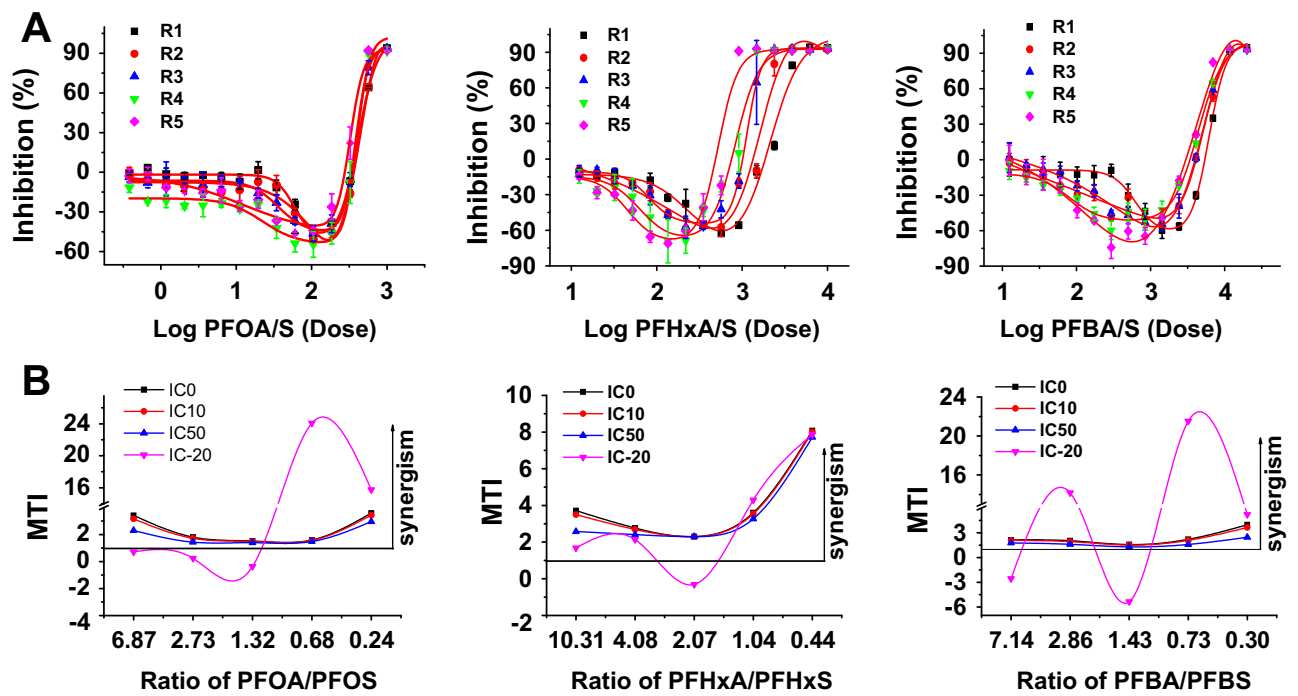

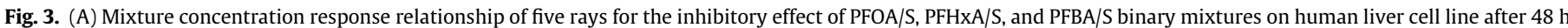

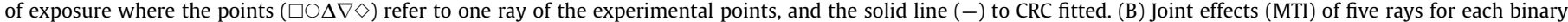

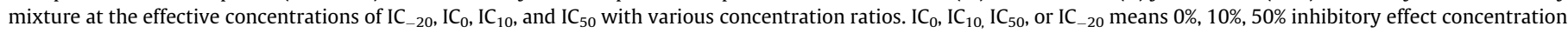
or $20 \%$ stimulatory effect concentration in the mixture.

Table 2

Concentration-response models of PFC mixtures and their joint effects on human liver cell line at $48 \mathrm{~h}$.

\begin{tabular}{|c|c|c|c|c|c|c|c|c|c|c|c|}
\hline & \multirow[t]{2}{*}{$\mathrm{IC}_{-20}$} & \multirow[t]{2}{*}{$\mathrm{IC}_{0}$} & \multirow[t]{2}{*}{$\mathrm{IC}_{10}$} & \multirow[t]{2}{*}{$\mathrm{IC}_{50}$} & \multirow[t]{2}{*}{$M$} & \multicolumn{2}{|l|}{ TU } & \multicolumn{2}{|l|}{$\mathrm{AI}$} & \multicolumn{2}{|l|}{ MTI } \\
\hline & & & & & & $M_{0}$ & Effect & $\mathrm{AI}$ & Effect & MTI & Effect \\
\hline${ }^{\mathrm{a}} \mathrm{Mix}_{0} 11$ & $7.50 \times 10-5$ & $2.69 \times 10^{-3}$ & $2.49 \times 10^{-3}$ & $3.61 \times 10^{-3}$ & 1.32 & 9.83 & Partial addition & -0.32 & Antagonism & 0.88 & Partial addition \\
\hline${ }^{\mathrm{a}} \operatorname{Mix}_{10} 11$ & $9.67 \times 10^{-5}$ & $1.99 \times 10^{-3}$ & $1.81 \times 10^{-3}$ & $2.80 \times 10^{-3}$ & 1.04 & 10.27 & Partial addition & -0.04 & Antagonism & 0.98 & Partial addition \\
\hline${ }^{\mathrm{a}} \mathrm{Mix}_{50} 11$ & $7.44 \times 10^{-5}$ & $2.16 \times 10^{-3}$ & $1.93 \times 10^{-3}$ & $3.16 \times 10^{-3}$ & 1.41 & 10.47 & Partial addition & -0.41 & Antagonism & 0.85 & Partial addition \\
\hline${ }^{\mathrm{b}} \mathrm{Mix}_{0} 9$ & $3.39 \times 10^{-5}$ & $1.19 \times 10^{-3}$ & $1.29 \times 10^{-3}$ & $1.79 \times 10^{-3}$ & 0.57 & 9.00 & Synergism & 0.74 & Synergism & 1.25 & Synergism \\
\hline${ }^{\mathrm{b}} \mathrm{Mix}_{10} 9$ & $4.16 \times 10^{-5}$ & $1.41 \times 10^{-3}$ & $1.53 \times 10^{-3}$ & $2.09 \times 10^{-3}$ & 0.70 & 9.00 & Synergism & 0.43 & Synergism & 1.16 & Synergism \\
\hline${ }^{\mathrm{b}} \mathrm{Mix}_{50} 9$ & $8.78 \times 10^{-5}$ & $1.29 \times 10^{-3}$ & $1.40 \times 10^{-3}$ & $1.89 \times 10^{-3}$ & 0.69 & 9.00 & Synergism & 0.44 & Synergism & 1.17 & Synergism \\
\hline${ }^{\mathrm{b}} \mathrm{Mix}_{-20} 9$ & $3.03 \times 10^{-4}$ & $3.14 \times 10^{-3}$ & $3.42 \times 10^{-3}$ & $4.76 \times 10^{-3}$ & 1.36 & 9.00 & Partial addition & -0.36 & Antagonism & 0.86 & Partial addition \\
\hline${ }^{\mathrm{c}} \mathrm{Mix}_{-20} 7$ & $1.81 \times 10^{-5}$ & $5.47 \times 10^{-4}$ & $5.76 \times 10^{-4}$ & $7.27 \times 10^{-4}$ & 0.61 & 7.00 & Synergism & 0.65 & Synergism & 1.26 & Synergism \\
\hline
\end{tabular}

TU: toxic units, $M=\sum \mathrm{TU}_{\mathrm{i}} ; M_{0}=M / \max \left(\mathrm{TU}_{\mathrm{i}}\right)$; $\mathrm{AI}$ : additive index; MTI: mixture toxic index.

a Eleven PFAAs (PFBA, PFHxA, PFOA, PFNA, PFDA, PFUnDA, PFDoDA, PFTdDA, PFBS, PFHxS, and PFOS).

b Nine PFAAs (PFBA, PFHxA, PFOA, PFNA, PFDA, PFUnDA, PFBS, PFHxS, and PFOS).

c Seven PFAAs (PFOA, PFNA, PFDA, PFUnDA, PFBS, PFHxS, and PFOS).

also chosen to determine if similar interactions were taking place between PFSA and its corresponding PFCA mixture. Overall, three binary mixtures with $\mathrm{J}$-shaped concentration-response curves were observed (Fig. 3A). The fitted CRC models and resulting parameters for the binary mixtures are listed in Table S3 and the fitted models and details of the experiment are given in the supporting information (Table S4). All $\mathrm{IC}_{0}, \mathrm{IC}_{10}$, and $\mathrm{IC}_{50}$ values in the three binary mixtures were less than that of individual PFSA or PFCA, respectively. The index values of M, AI, and MTI for the binary mixtures of PFSA and PFCA implied that a synergistic action took place under the effective concentrations of $\mathrm{IC}_{0}, \mathrm{IC}_{10}$, and $\mathrm{IC}_{50}$ with various concentration ratios (Fig. $3 \mathrm{~B}$ and Fig. S2). For the three binary mixtures, PFHxA/S showed the strongest synergistic effect with MTI values from 2.30 to 8.08 for five rays, followed by PFOA/S with MTI values from 1.40 to 3.59 for five rays. For the effective concentration of IC -20 , however, the joint effects were quite different among the three binary mixtures (PFBA/S, PFHxA/ $\mathrm{S}$, and PFOA/S). Only the higher proportion of PFSA in the mixtures (PFOS $>59.7 \%$, PFHXS $>48.9 \%$, and PFBS $>57.7 \%$, respectively) showed a synergistic effect for IC $_{-20}$. For the effective concentration of $\mathrm{IC}_{-20}$ in the PFSA/PFCA mixture, the strongest synergistic effect of the binary mixture was observed with a MTI value of 24.09 at 0.68 for the ratio of PFOA/PFOS.

The mixture of PFHxS and PFHxA showed the highest synergistic effect among the three mixtures, especially at the high proportion of PFHxS (70\%), and indicated that PFHxS had the highest contribution to the synergistic effect.

\subsection{Non-monotonic concentration-response relationships of multiple PFAA mixtures}

We exposed HL-7702 cells to mixtures of eleven and nine individual PFAAs to determine the joint effects of PFAA mixtures. In the mixtures, the concentration ratios of various PFAAs were the ratios of their individual $\mathrm{IC}_{x}$ to the total concentration of the mixtures (Tables S5 and S6). Two sets of mixtures were composed of the eleven individual PFAAs (C4-C14) with both J-shaped and S-shaped curves, while the mixtures of the nine individual PFAAs with Jshaped curves only (C4-C11). Resulting parameters for the fitted CRC models are listed in Table 2 and the fitted models and details are given in Table S7. All PFAA mixtures presented non-monotonic J-shaped CRCs (Fig. 4A and Fig. S3A). 

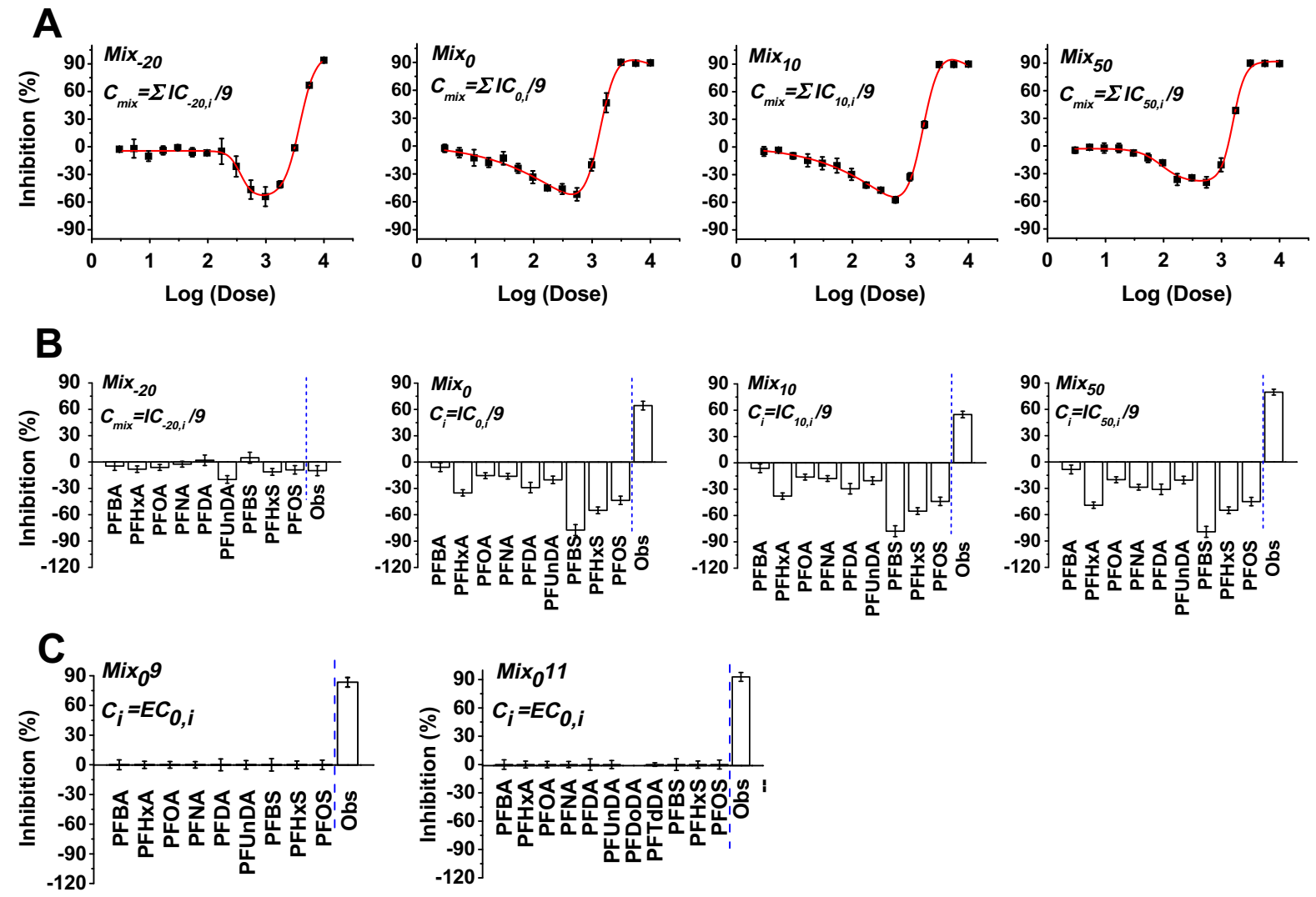

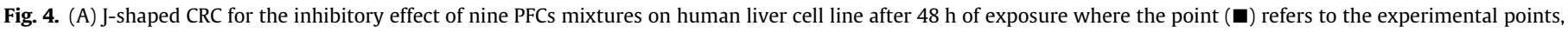

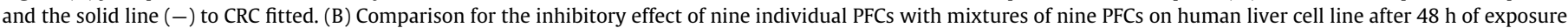

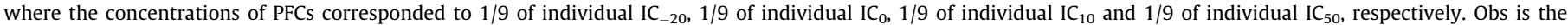

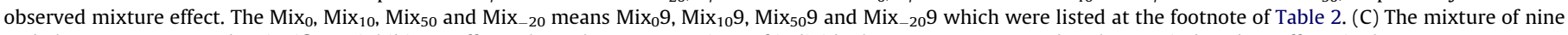
and eleven PFAAs caused a significant inhibitory effect where the concentrations of individual PFAAs were IC ${ }_{0, i}$ and each PFAA induced no effect singly.

To test the hypothesis that a $n$-component mixture, whose concentration equals the sum of $\mathrm{IC}_{x, i} / n$ of the individual components, will exactly produce an effect of $x \%$, we calculated the effect of individual PFAAs at concentrations of $\mathrm{IC}_{0, i} / n, \mathrm{IC}_{10, i} / n$, and $\mathrm{IC}_{50, i} / n(n=9$ or 11) based on their individual CRCs (Fig. 1). The results of the mixtures $\left(\mathrm{Mix}_{0}, \mathrm{Mix}_{10}\right.$, and $\left.\mathrm{Mix}_{50}\right)$ of the eleven PFAAs showed that each PFAA at concentrations of $\mathrm{IC}_{-x}, \mathrm{l} / 11$ induced a stimulatory effect singly and the mixture at the concentration of $\Sigma \mathrm{IC}_{-x} / 11$ did not produce an inhibitory effect (Fig. S3B). However, the concentrations of individual PFAAs for $\mathrm{IC}_{0, i} / 9, \mathrm{IC}_{10, i} / 9$, and $\mathrm{IC}_{50, i} / 9$ with $\mathrm{J}-$ shaped curves only induced a stimulatory effect singly; the three mixtures $\left(\mathrm{Mix}_{0}, \mathrm{Mix}_{10}\right.$, and $\left.\mathrm{Mix}_{50}\right)$ at concentrations of $\Sigma \mathrm{IC}_{0} / 9$, $\Sigma \mathrm{IC}_{10} / 9$, and $\Sigma \mathrm{IC}_{50} / 9$ produced significant inhibitory effects based on each mixture's CRCs (Fig. 4B).

In addition, the mixture with $\mathrm{IC}_{0, i}$ for the concentration of individual PFAA caused a significant inhibitory effect; however, no PFAA at $\mathrm{IC}_{0, i}$ concentration induced an inhibitory effect singly (Fig. 4C).

As it is not possible to determine $\mathrm{IC}_{0}$ from a typical S-shaped $\mathrm{CRC}$, no-observed-effect concentration (NOEC) can be regarded as an approximation of $\mathrm{IC}_{0}$. Certain evidence has indicated that adverse effects of mixtures could be induced from exposure to many compounds at or below NOEC (Silva et al., 2002; Rajapakse et al., 2002; Ge et al., 2011). We assessed the joint effects of the eleven and nine individual PFAAs at $\mathrm{IC}_{0}$ concentration corresponding to a true zero effective concentration in our test system. The $\mathrm{IC}_{0}$ mixtures $\left(\mathrm{Mix}_{0} 11\right.$ and $\left.\mathrm{Mix}_{0} 9\right)$ for the eleven and nine individual PFAAs produced a $92.86 \%$ and $83.47 \%$ inhibitory effect on human liver cell line, respectively. Our results implied that the individual stimulatory responses were cumulative and produced adverse effects in mixtures at low dose levels. This finding also demonstrated that the $\mathrm{IC}_{0}$ value of individual chemicals should not be regarded as a safe level in risk assessment of environmental mixtures.

All three mixtures $\left(\mathrm{Mix}_{0}, \mathrm{Mix}_{10}\right.$, and $\mathrm{Mix}_{50}$ ) of the eleven PFAAs presented a partial addition effect as evaluated by Toxic Unit (TU), Additive Index (AI), and Mixture Toxicity Index (MTI) (Table 2). We next chose nine individual PFAAs with J-shaped curves only (C4C11) to study the four concentration effects of EECR mixtures $\left(\mathrm{Mix}_{-20}, \mathrm{Mix}_{0}, \mathrm{Mix}_{10}\right.$, and $\left.\mathrm{Mix}_{50}\right)$. All Mix, $\mathrm{Mix}_{10}$, and $\mathrm{Mix}_{50} \mathrm{mix}-$ tures presented a synergistic effect with MTI values ranging from 1.16 to 1.25 . However, the mixtures of $\mathrm{Mix}_{-20}$ for nine individual PFAAs showed partial addition. Since the concentrations of IC -20 for PFBA and PFHxA were much higher than other PFAAs, we next excluded them to form another mixture set of seven individual PFAAs. The results showed that a synergistic effect took place, and the MTI values of the mixtures for $\mathrm{Mix}_{-20}$ of nine and seven individual PFAAs were 0.86 and 1.26 , respectively (Table 2 ). This indicated that the composition of mixture with the parallel CRCs may have similar mechanisms and behave synergistically. Our research provides evidence that synergistic or partial addition effects of PFAAs in the human body may occur.

In conclusion, individual and mixtures of PFAAs (C4-C11) had an obvious non-monotonic concentration-response relationship on human liver cells. Results of the three binary mixtures of PFAAs showed that synergistic effects occurred under effective concentrations of $\mathrm{IC}_{0}, \mathrm{IC}_{10}$, and $\mathrm{IC}_{50}$ in mixtures while under IC -20 the synergistic effect only occurred under a higher proportion of PFSA. The EECR mixtures of nine individual PFAAs with non-monotonic 
J-shaped curves showed synergistic effects. Our results implied that the individual stimulatory responses to PFAA at relevant dose levels may produce adverse effects in mixtures.

\section{Acknowledgements}

This work was supported by the National Basic Research Program of China (973; Grant: 2009CB118802) and the National Natural Science Foundation of China (Grants 31025006 and 21077126).

\section{Appendix A. Supplementary material}

Supplementary data associated with this article can be found, in the online version, at http://dx.doi.org/10.1016/j.chemosphere.2013. 07.033.

\section{References}

Albrecht, P.P., Torsell, N.E., Krishnan, P., Ehresman, D.J., Frame, S.R., Chang, S.C., et al. 2013. A species difference in the peroxisome proliferator-activated receptor $\alpha$ dependent response to the developmental effects of perfluorooctanoic acid. Toxicological Sciences 131, 568-582.

Apelberg, B.J., Witter, F.R., Herbstman, J.B., Calafat, A.M., Halden, R.U., Needham, L.L., et al., 2007. Cord serum concentrations of perfluorooctane sulfonate (PFOS) and perfluorooctanoate (PFOA) in relation to weight and size at birth. Environmental Health Perspectives 115, 1670-1676.

Berridge, M.V., Herst, P.M., Tan, A.S., 2005. Tetrazolium dyes as tools in cell biology: new insights into their cellular reduction. Biotechnology Annual Review 11, $127-152$.

Bjork, J.A., Wallace, K.B., 2009. Structure-activity relationships and human relevance for perfluoroalkyl acid-induced transcriptional activation of peroxisome proliferation in liver cell cultures. Toxicological Sciences 111, 89-99.

Buck, R.C., Franklin, J., Berger, U., Conder, J.M., Cousins, I.T., de Voogt, P., et al., 2011. Perfluoroalkyl and polyfluoroalkyl substances in the environment: terminology, classification, and origins. Integrated Environmental Assessment Management 7, 513-541.

Conder, J.M., Hoke, R.A., Wolf, W.D., Russell, M.H., Buck, R.C., 2008. Are PFCAs Bioaccumulative A Critical Review and Comparison with Regulatory Criteria and Persistent Lipophilic Compounds. Environmental Science and Technology 42, 995-1003.

De Silva, A.O., Mabury, S.A., 2006. Isomer distribution of perfluorocarboxylates in human blood: potential correlation to source. Environmental Science and Technology 40, 2903-2909.

Dou, R.N., Liu, S.S., Mo, L.Y., Liu, H.L., Deng, F.C., 2011. A novel direct equipartition ray design (EquRay) procedure for toxicity interaction between ionic liquid and dichlorvos. Environmental Science and Pollution Research 18, 734-742.

Filipovic, M., Berger, U., McLachlan, M.S., 2013. Mass balance of perfluoroalkyl acids in the baltic sea. Environmental Science and Technology 47, 4088-4095.

Gallo, V., Leonardi, G., Genser, B., Lopez-Espinosa, M., Frisbee, S.G., Karlsson, L., et al., 2012. Serum perfluorooctanoate (PFOA) and perfluorooctane sulfonate (PFOS) concentrations and liver function biomarkers in a population with elevated PFOA exposure. Environmental Health Perspectives 120, 655-660.

Ge, H.L., Liu, S.S., Zhu, X.W., Liu, H.L., Wang, L.J., 2011. Predicting hormetic effects of ionic liquid mixtures on luciferase activity using the concentration addition model. Environmental Science and Technology 45, 1623-1629.

Guida, M., Borriello, I., Gallo, M., Castello, G., Pagano, G., 2008. Complex mixtureassociated hormesis and toxicity: The case of leather tanning industry. DoseResponse 6, 383-396.

Houde, M., Martin, J.W., Letcher, R.J., Solomon, K.R., Muir, D.C., 2006. Biological monitoring of polyfluoroalkyl substances: A review. Environmental Science and Technology 40, 3463-3473.

Kannan, K., Corsolini, S., Falandysz, J., Fillmann, G., Kumar, K.S., Loganathan, B.G., et al., 2004. Perfluorooctanesulfonate and related fluorochemicals in human blood from several countries. Environmental Science and Technology 38, 44894495.

Kärrman, A., Ericson, I., van Bavel, B., Darnerud, P.O., Aune, M., Glynn, A., et al., 2006. Exposure of perfluorinated chemicals through lactation: Levels of matched human milk and serum and a temporal trend, 1996-2004, in Sweden. Environmental Health Perspectives 115, 226-230.

Keiter, S., Baumann, L., Färber, H., Holbech, H., Skutlarek, D., Engwall, M., et al., 2012. Long-term effects of a binary mixture of perfluorooctane sulfonate (PFOS) and bisphenol A (BPA) in zebrafish (Danio rerio). Aquat Toxicology 118-119, 116 129.

Koutsaftis, A., Aoyama, I., 2007. Toxicity of four antifouling biocides and their mixtures on the brine shrimp Artemia salina. Science of the Total Environment $387,166-174$.

Kowalczyk, J., Ehlers, S., Fürst, P., Schafft, H., Lahrssen-Wiederholt, M., 2012 Transfer of perfluorooctanoic acid (PFOA) and perfluorooctane sulfonate (PFOS) from contaminated feed into milk and meat of sheep: pilot study. Archives of Environmental Contamination and Toxicology 63, 288-298.

Lau, C., Butenhoff, J.L., Rogers, J.M., 2004. The developmental toxicity of perfluoroalkyl acids and their derivatives. Toxicology and Applied Pharmacology 198, 231-241.

Li, F., Xie, Q., Li, X.H., Li, N., Chi, P., Chen, J.W., et al., 2010. Hormone activity of hydroxylated polybrominated diphenyl ethers on human thyroid receptor-beta: In vitro and in silico investigations. Environmental Health Perspectives 118, 602-606.

Liu, S.S., Zhang, J., Zhang, Y.H., Qin, L.T., 2012. APTox: assessment and prediction on toxicity of chemical mixtures. Acta Chimica Sinica 70, 1511-1517.

Olsen, G.W., Huang, H.Y., Helzlsouer, K.J., Hansen, K.J., Butenhoff, J.L., Mandel, J.H., 2005. Historical comparison of perfluorooctanesulfonate, perfluorooctanoate, and other fluorochemicals in human blood. Environmental Health Perspectives $113,539-545$.

Rajapakse, N., Silva, E., Kortenkamp, A., 2002. Combining xenoestrogens at levels below individual no-observed-effect concentrations dramatically enhances steroid hormone action. Environmental Health Perspectives 110, 917-921.

Renner, R., 2001. Growing concern over perfluorinated chemicals. Environmental Science and Technology 35, 154A-160A.

Seacat, A.M., Thomford, P.J., Hansen, K.J., Clemen, L.A., Eldridge, S.R., Elcombe, C.R., et al., 2003. Sub-chronic dietary toxicity of potassium perfluorooctanesulfonate in rats. Toxicology 183, 117-131.

Silva, E., Rajapakse, N., Kortenkamp, A., 2002. Something from "nothing"-eight weak estrogenic chemicals combined at concentrations below NOECs produce significant mixture effects. Environmental Science and Technology 36, 17511756.

Son, H.Y., Kim, S.H., Shin, H.I., Bae, H.I., Yang, J.H., 2008. PerXuorooctanoic acidinduced hepatic toxicity following 21-day oral exposure in mice. Archives of Toxicology 82, 239-246.

Steenland, K., Tinker, S., Frisbee, S., Ducatman, A., Vaccarino, V., 2009. Association of perfluorooctanoic acid and perfluorooctane sulfonate with serum lipids among adults living near a chemical plant. American Journal of Epidemiology 170, 1268-1278.

Wang, F., Liu, W., Jin, Y., Dai, J., Zhao, H., Xie, Q., et al., 2011. Interaction of PFOS and BDE-47 co-exposure on thyroid hormone levels and TH-related gene and protein expression in developing rat brains. Toxicological Sciences 121, 279291.

Weiss, J.M., Andersson, P.L., Lamoree, M.H., Leonards, P., van Leeuwen, S., Hamers, T., 2009. Competitive binding of poly- and perfluorinated compounds to the thyroid hormone transport protein transthyretin. Toxicological Sciences 109, 206-216.

Wolf, D.C., Moore, T., Abbott, B.D., Rosen, M.B., Das, K.P., Zehr, R.D., et al., 2008 Comparative hepatic effects of perfluorooctanoic acid and WY 14,643 in PPAR- $\alpha$ knockout and wild-type mice. Toxicologic Pathology 36, 632-639.

$\mathrm{Xu}, \mathrm{S}$., Nirmalakhandan, N., 1998. Use of QSAR models in predicting joint effects in multi-component mixtures of organic chemicals. Water Research 32, 23912399.

Zhang, H.X., Shi, Z.M., Liu, Y., Wei, Y.H., Dai, J.Y., 2008. Lipid homeostasis and oxidative stress in the liver of male rats exposed to perfluorododecanoic acid. Toxicology and Applied Pharmacology 227, 16-25.

Zhang, W., Liu, Y., Zhang, H.X., Dai, J.Y., 2012. Proteomic analysis of male zebrafish livers chronically exposed to perfluorononanoic acid. Environment International 42, 20-30.

Zhao, C.Y., Boriani, E., Chana, A., Roncaglioni, A., Benfenati, E., 2008. A new hybrid system of QSAR models for predicting bioconcentration factors (BCF). Chemosphere 73, 1701-1707.

Zhu, X.W., Liu, S.S., Ge, H.L., Liu, Y., 2009. Comparision between two confidence intervals of dose-response relationships. China Environmental Science 29, 113117. 